\title{
A FAST APPROACH FOR THE LOG-CUMULANTS METHOD APPLIED TO INTENSITY SAR IMAGE PROCESSING
}

\author{
F. A. A. Rodrigues ${ }^{1}$, J. S. Nobre ${ }^{2}$, R. Vigélis ${ }^{3}$, V. Liesenberg $^{4}$, R. C. P. Marques ${ }^{5}$, F. N. S. Medeiros ${ }^{6}$, \\ ${ }^{1}$ Federal University of Cariri, Juazeiro do Norte, Brazil- alixandre.avila@ufca.edu.br \\ ${ }^{2}$ Department of Statistics and Applied Mathematics, Federal University of Ceara, Fortaleza, Brazil - juvencio@ufc.br \\ ${ }^{3}$ Federal University of Ceara, Sobral, Brazil - rfvigelis@gmail.com \\ ${ }^{4}$ Santa Catarina State University, Santa Catarina, Brazil - veraldo@ gmail.com \\ ${ }^{5}$ Federal Institute of Education, Science and Tecnology of Ceara, Fortaleza, Brazil - regismarques@ifce.edu.br \\ ${ }^{6}$ Teleinformatics Engineering Department, DETI-Centro de Tecnologia, Cx. Postal 6007, Campus do Pici, s/n, Fortaleza, CE, \\ Brazil-fsombra@ufc.br
}

KEY WORDS: SAR image, log-cumulants, parameter estimator, change detection.

\begin{abstract}
:
Synthetic aperture radar (SAR) image processing and analysis rely on statistical modeling and parameter estimation of the probability density functions that characterize data. The method of log-cumulants (MoLC) is a reliable alternative for parameter estimation of SAR data models and image processing. However, numerical methods are usually applied to estimate parameters using MoLC, and it may lead to a high computational cost. Thus, MoLC may be unsuitable for real-time SAR imagery applications such as change detection and marine search and rescue, for example. Our paper introduces a fast approach to overcome this limitation of MoLC, focusing on parameter estimation of single-channel SAR data modeled by the $G_{I}^{0}$ distribution. Experiments with simulated and real SAR data demonstrate that our approach performs faster than MoLC, while the precision of the estimation is comparable with that of the original MoLC. We tested the fast approach with multitemporal data and applied the arithmetic-geometric distance to real SAR images for change detection on the ocean. The experiments showed that the fast MoLC outperformed the original estimation method with regard to the computational time.
\end{abstract}

\section{INTRODUCTION}

The processing and analysis of synthetic aperture radar (SAR) images are relevant for several remote sensing applications such as the monitoring of natural features and change detection on the Earth under certain weather conditions (López-Martínez, Fàbreagas, 2003), among other applications. Appropriate statistical modeling that can accurately describe synthetic aperture radar (SAR) data plays a central role in the interpretation of SAR images (Frery et al., 1997). SAR image processing algorithms that rely on statistical models usually require parameter estimation. The method of log-cumulants (MoLC) is appropriate for parameter estimation of probability density functions that describe SAR data (Bujor et al., 2004), and it is well suited to a wide range of image processing algorithms and SAR applications (Bujor et al., 2004), (Krylov et al., 2013), (Rodrigues et al., 2016).

The probability density function named $G_{I}^{0}$ distribution (Frery et al., 1997) characterizes intensity SAR images and it is defined over $\mathbb{R}^{+}$. According to (Krylov et al., 2013), the use of the Mellin transform makes it possible to perform a more effective analysis of practically important distributions defined in $\mathbb{R}^{+}$. Thus, MoLC is a suitable option for parameter estimation of the $G_{I}^{0}$ distribution. MoLC has previously been applied to SAR images, and especially to small samples, which pose a critical issue in many applications (Krylov et al., 2013). Algorithms for SAR image processing (Rodrigues et al., 2016), classification (Singh, Datcu, 2013) and change detection with multitemporal SAR images (Bujor et al., 2004) have successfully used parameters of probability density functions estimated by MoLC as inputs. However, the parameter estimation of the $G_{I}^{0}$ distribution using MoLC adopts numerical solution procedures which usually lead to higher computational cost.

Based on the definitions of the polygamma functions and gamma function (Arfken, Weber, 2005), we introduce an analytical formulation to lessen the computational time of the log-cumulants method to estimate the roughness and scale parameters of the $G_{I}^{0}$ distribution. These estimates can be inputs to algorithms for intensity SAR images processing and classification.

Based on the definitions of the polygamma functions and gamma function (Arfken, Weber, 2005), our proposed fast formulation for MoLC considerably speeds up the parameter estimation of the $G_{I}^{0}$ distribution. To evaluate the performance of the fast formulation, we performed experiments on synthetic and real SAR data to compare it with the original MoLC in terms of the computational time and estimation accuracy.

\section{MATERIALS AND METHODS}

\subsection{The log-cumulants method}

Let $Z$ be a continuous random variable with the probability density function $f_{Z}(z, \theta)$ defined over $\mathbb{R}^{+}$, where $\theta$ can be either a real-valued parameter or a vector. The derivation of MoLC applies the Mellin transform to $f_{Z}(z, \theta)$, which yields:

$$
\phi_{Z}(s)=\int_{0}^{\infty} z^{s-1} f_{Z}(z, \theta) \mathrm{d} z=E\left[Z^{s-1}\right]
$$


where $E\left[Z^{s-1}\right]$ is the moment of order $s-1$ of the random variable $Z$.

The log-moment and log-cumulant of order $v$ are two important relations used to formulate MoLC. To obtain the analytical expressions of the $\log$-moment and $\log$-cumulant of order $v$, we differentiate $\phi_{Z}(s)$ and evaluate it at $s=1$. The log-moment of order $v$ is given by (Nicolas, 2002):

$$
\widetilde{m}_{v}=\left.\frac{\mathrm{d}^{v} \phi_{z}(s)}{\mathrm{d} s^{v}}\right|_{s=1}, v \in \mathbb{N}^{*}
$$

Based on the natural logarithm of $\phi_{Z}(s)$, the log-cumulant of order $v$ is expressed by (Nicolas, 2002):

$$
\widetilde{k}_{v}=\left.\frac{\mathrm{d}^{v} \psi_{z}(s)}{\mathrm{d} s^{v}}\right|_{s=1}, v \in \mathbb{N}^{*}
$$

with $\psi_{z}(s)=\ln \left(\left(\phi_{z}(s)\right)\right.$.

The strategy used in MoLC to estimate $\theta$ is based on the relation between the log-moments and log-cumulants. The logcumulants can be obtained from the log-moments using the relation:

$$
\left\{\begin{array}{l}
\widetilde{k}_{1}=\widetilde{m}_{1} \\
\widetilde{k}_{v}=\widetilde{m}_{v}-\sum_{i=1}^{v-1}\left(\begin{array}{c}
v-1 \\
i-1
\end{array}\right) \widetilde{k}_{i} \widetilde{m}_{v-i}, v \in\{2,3 \ldots\} .
\end{array}\right.
$$

Thus, for example, the log-cumulants and log-moments of order 1 and 2 are related by:

$$
\left\{\begin{array}{l}
\widetilde{k}_{1}=\widetilde{m}_{1} \\
\widetilde{k}_{2}=\widetilde{m}_{2}-\widetilde{m}_{1}^{2} .
\end{array}\right.
$$

Estimation of the parameter vector $\theta$ is generally achieved by replacing $\widetilde{m}_{v}$ by the corresponding sample log-moment, which is given by (Nicolas, 2002):

$$
\widehat{\widetilde{m}}_{v}=\frac{1}{n} \sum_{i=1}^{n} \ln z_{i}^{v}
$$

with $z_{i}, i \in\{1,2, \ldots, n\}$, being a sample of a random variable $Z$.

\subsection{The log-cumulants method for the $G_{I}^{0}$ distribution}

The $G_{I}^{0}$ continuous distribution, with parameter vector $\theta=$ $(\alpha, \gamma, L)^{\top}$, is characterized by the probability density function (Frery et al., 1997):

$$
f_{G_{I}^{0}}(z, \theta)=\frac{L^{L} \Gamma(L-\alpha)}{\gamma^{\alpha} \Gamma(-\alpha) \Gamma(L)} z^{L-1}(\gamma+L z)^{\alpha-L},
$$

where $z>0, \alpha<0$ stands for the roughness parameter, $\gamma>0$ is the scale parameter, $L \geq 1$ is the number of looks and $\Gamma($.) corresponds to the gamma function. The $r$ th-order moment for the $G_{I}^{0}$ distribution is defined as follows:

$$
E_{G_{I}^{0}}\left[Z^{r}\right]=\left(\frac{\gamma}{L}\right)^{r} \frac{\Gamma(-\alpha-r) \Gamma(L+r)}{\Gamma(-\alpha) \Gamma(L)}
$$

with $\alpha<-r$.

For the probability density function in Equation (7), the Mellin transform $\phi_{Z}(s)$ can be obtained using Equations (1) and (8), giving rise to the Mellin transform for the $G_{I}^{0}$ distribution:

$$
\phi_{G_{I}^{0}}(s)=\left(\frac{\gamma}{L}\right)^{s-1} \frac{\Gamma(1-s-\alpha) \Gamma(L+s-1)}{\Gamma(-\alpha) \Gamma(L)} .
$$

In this paper, for the sake of simplicity, the number of looks $L$ is assumed to be known. Thus, we obtain the log-cumulants of order 1 and 2 for the $G_{I}^{0}$ distribution using Equation (3):

$$
\left\{\begin{array}{l}
\widetilde{k}_{1}=\ln \left(\frac{\gamma}{L}\right)+\Psi^{0}(L)-\Psi^{0}(-\alpha) \\
\widetilde{k}_{2}=\Psi^{1}(L)+\Psi^{1}(-\alpha),
\end{array}\right.
$$

where $\Psi^{0}($.$) and \Psi^{1}($.$) are the digamma and trigamma func-$ tions (Arfken, Weber, 2005), respectively.

To estimate the parameter vector $\theta^{*}=(\alpha, \gamma)^{\top}$, we apply Equations (5) and (10) and replace $\widetilde{m}_{1}$ and $\widetilde{m}_{2}$ by the corresponding sample log-moments in Equation (6).

Given that there are no feasible inverse functions for $\Psi^{0}($.$) and$ $\Psi^{1}($.$) , the non-linear system in Equation (10) cannot be solved$ explicitly and therefore it requires iterative procedures. Overall, these procedures are computationally slow, and hence a key challenge is to reduce this computational cost.

\subsection{Fast approach for log-cumulants method}

This section introduces our fast approach to the method of logcumulants (FAMoLC) to estimate the vector of parameters, $(\alpha, \gamma)^{\top}$, of the $G_{I}^{0}$ distribution. To solve the non-linear system in Equation (10), we use the definition of the polygamma function (Arfken, Weber, 2005):

$$
\Psi^{n}(w)=\frac{d^{n+1} \ln (\Gamma(w))}{d w^{n+1}},
$$

where $n \in \mathbb{N}^{*}$ and $w>0$. The gamma function $\Gamma(w)$ in Equation (11) can be defined as (Arfken, Weber, 2005):

$$
\Gamma(w)=\frac{1}{w e^{\tau w} \prod_{k=1}^{\infty}\left(1+\frac{w}{k}\right) e^{-\frac{w}{k}}}
$$

where $\tau$ is the Euler-Macheroni constant. Using Equation (12), $\ln (\Gamma(w))$ in Equation (11) can be rewritten as:

$$
\ln (\Gamma(w))=-\ln (w)-\tau w-\sum_{k=1}^{\infty}\left[\ln \left(1+\frac{w}{k}\right)-\frac{w}{k}\right]
$$


According to Equation (11), $\Psi^{1}(-\alpha)$ in the non-linear system of Equation (10) is given by:

$$
\Psi^{1}(-\alpha)=\frac{d^{2} \ln (\Gamma(-\alpha))}{d(-\alpha)^{2}} .
$$

To achieve the partial derivatives of $\Psi^{1}(-\alpha)$ in Equation (14) and considering that $\ln (\Gamma(-\alpha))$ can be rewritten using Equation (13), we compute $\Psi^{1}(-\alpha)$ as:

$$
\Psi^{1}(-\alpha)=\frac{1}{(-\alpha)^{2}}+\sum_{k=1}^{\infty} \frac{1}{(k-\alpha)^{2}} .
$$

Denoting the infinite sum in Equation (15) by $\xi(\alpha)$ and considering that $(-\alpha)^{2}=\alpha^{2}$, we have:

$$
\Psi^{1}(-\alpha)=\frac{1}{\alpha^{2}}+\xi(\alpha)
$$

Our assumption is that $\left(\frac{1}{\alpha^{2}}\right)$ in Equation (16) is an approximation of $\Psi^{1}(-\alpha)$ :

$$
\Psi^{1}(-\alpha) \approx \frac{1}{\alpha^{2}}
$$

Here, we employ the approximation in Equation (17) to rewrite Equation (10) and then estimate the roughness parameter with the following analytic expression:

$$
\hat{\alpha}= \begin{cases}-\mid \sqrt{\frac{1}{\widetilde{k}_{2}-\Psi^{1}(L)} \mid, \text { if }} \quad \widetilde{k}_{2}-\Psi^{1}(L)<0 \\ -\sqrt{\frac{1}{\widetilde{k}_{2}-\Psi^{1}(L)}, \text { if }} \quad \widetilde{k}_{2}-\Psi^{1}(L)>0\end{cases}
$$

where |.| stands for the modulus operator and the first expression of Equation (18), $\sqrt{\frac{1}{\widetilde{k}_{2}-\Psi^{1}(L)}}$, yields a complex-valued result.

The proposed approach for the roughness parameter estimation is restricted by the condition $\Psi^{1}(L)-\widetilde{k}_{2}=0$, where there is no estimate. After obtaining $\widehat{\alpha}$, we estimate the scale parameter by placing $\widehat{\alpha}$ in the first term of Equation (10). Then, $\gamma$ can be estimated by:

$$
\hat{\gamma}=\exp \left[\widetilde{k}_{1}-\Psi^{0}(L)+\Psi^{0}(-\hat{\alpha})\right] L .
$$

Notice that we obtain the analytical expressions in Equations (18) and (19) because we discarded the term $\xi(\alpha)$ in Equation (16). Nevertheless, it is important to evaluate the magnitude of this term to compute $\Psi^{1}(-\alpha)$. Using Equation (16), the term $\xi(\alpha)$ can be rewritten as:

$$
\xi(\alpha)=\Psi^{1}(-\alpha)-\frac{1}{(-\alpha)^{2}}, \alpha<0 .
$$

The $\alpha$ parameter in the $G_{I}^{0}$ distribution is related to the roughness of a target. In (Gambini et al., 2015), the authors reported that $\alpha$ values close to zero (typically above -3 ) suggest extreme textured targets, regions with moderate texture usually produce $\alpha \in[-6,-3]$ and textureless targets $\alpha \in(-\infty,-6)$.
Fig. 1 illustrates that the magnitude of $\xi(\alpha)$ decreases with the increase of $|\alpha|$. Therefore, the proposed method provides better results in textureless targets, e.g. oil slicks on the ocean.

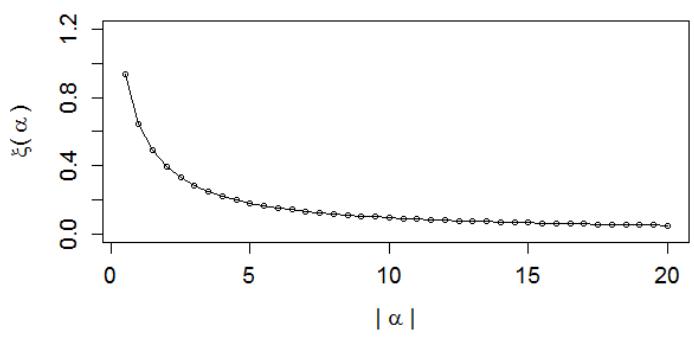

Figure 1. Approximation error.

\section{RESULTS AND DISCUSSSIONS}

To evaluate and compare the accuracy of the FAMoLC and MoLC, we performed a Monte Carlo experiment and computed the Mean-Square Error (MSE) for both parameter estimation methods. We also carried out experiments on multitemporal real SAR images to confirm the suitability of the fast approach for change detection on the ocean. Actually, the computational cost is a key point of surveillance systems. The experiments run on a $1.8 \mathrm{GHz}$ CPU with $8 \mathrm{~GB}$ of RAM.

\subsection{Experiments with simulated data}

The simulation of SAR data was based on the relation between intensity and amplitude data, in which $Z_{I}=Z_{A}^{2}$, as reported in (Frery et al., 1997) and (Lee, Pottier, 2009). Random samples of the random variable $Z_{I}$ following the $G_{I}^{0}$ distribution can be obtained using the expression

$$
Z_{I}=-\frac{\gamma}{\alpha} \Upsilon_{2 L,-2 \alpha}^{-1}(U)
$$

Here, $\Upsilon_{2 L,-2 \alpha}^{-1}$ stands for the inverse distribution function of $F$-Snedecor with $2 L$ and $-2 \alpha$ degrees of freedom, and $U$ a random variable with uniform distribution over $(0,1)$.

We assume distinct roughness parameter values $\alpha \in$ $\{-1.5,-3.0,-5.0\}$, number of looks $L \in\{1,5,8\}$ and without any loss of generality, we set the scale parameter $(\gamma)$ such that $E_{G_{I}^{0}}\left[Z_{I}\right]=1$, i.e., $\gamma=-\alpha-1$. Table 1 summarizes the scenarios used in the experiments with simulated data.

Table 1. The distinct scenarios for synthetic data simulation.

\begin{tabular}{cccccccccc}
\hline Scenario & $\mathbf{1}$ & $\mathbf{2}$ & $\mathbf{3}$ & $\mathbf{4}$ & $\mathbf{5}$ & $\mathbf{6}$ & $\mathbf{7}$ & $\mathbf{8}$ & $\mathbf{9}$ \\
\hline$\alpha$ & -1.5 & -1.5 & -1.5 & -3.0 & -3.0 & -3.0 & -5.0 & -5.0 & -5.0 \\
\hline$\gamma$ & 0.5 & 0.5 & 0.5 & 2 & 2 & 2 & 4 & 4 & 4 \\
\hline$L$ & 1 & 5 & 8 & 1 & 5 & 8 & 1 & 5 & 8 \\
\hline
\end{tabular}

We performed Monte Carlo experiments under different scenarios, where each one employed different sample sizes, i.e., $n \in\{9,25,49,81,121\}$. For each sample size, we performed 1.000 replicates per simulation yielding 45000 tests. The scale and roughness parameters of the synthetic data modeled by the 


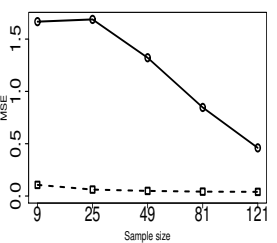

(a) Scenario 1

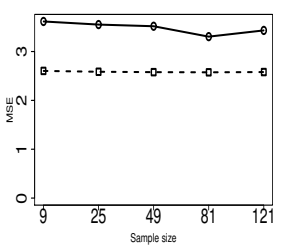

(d) Scenario 4

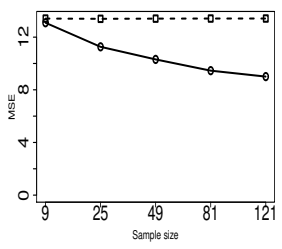

(g) Scenario 7

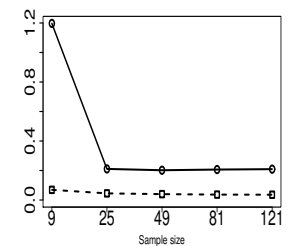

(b) Scenario 2

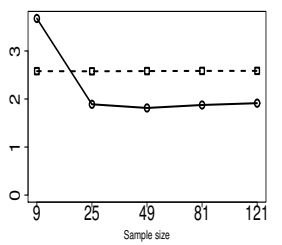

(e) Scenario 5

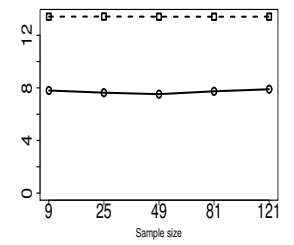

(h) Scenario 8

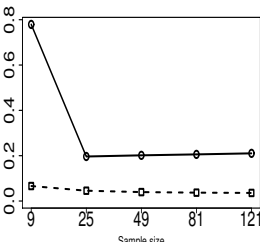

(c) Scenario 3

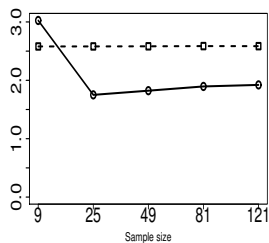

(f) Scenario 6

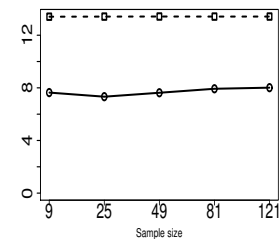

(i) Scenario 9

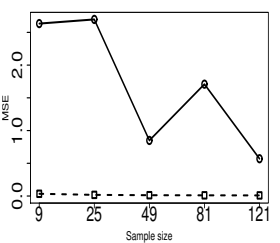

(a) Scenario 1

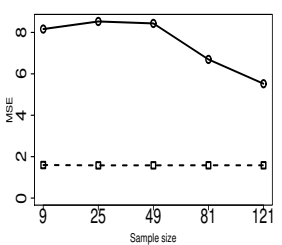

(d) Scenario 4

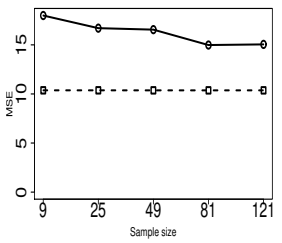

(g) Scenario 7

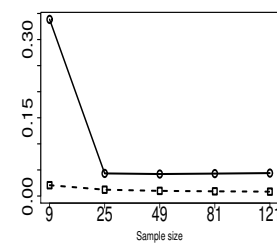

(b) Scenario 2

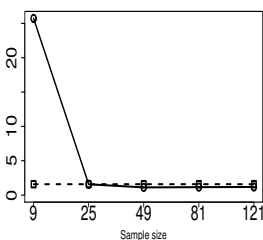

(e) Scenario 5

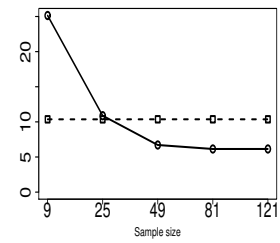

(h) Scenario 8

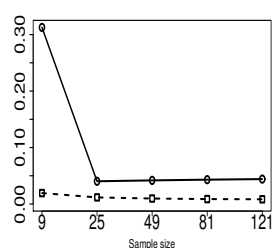

(c) Scenario 3

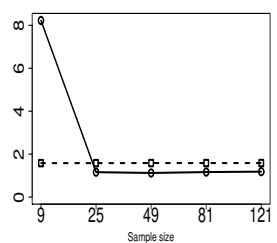

(f) Scenario 6

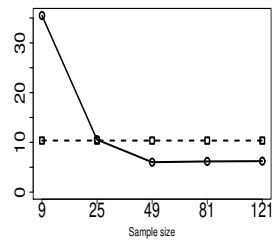

(i) Scenario 9

Figure 2. Mean-square error of the roughness parameter $(\alpha)$ estimates. The solid line refers to FMoLC and the dot line to MoLC.

$G_{I}^{0}$ were estimated using FAMoLC and MoLC. Fig. 2 and Fig. 3 illustrate the performance of both FAMoLC and MoLC, in terms of MSE, for estimates of $\alpha$ and $\gamma$.

The results indicated that FAMoLC was more sensitive to sample size than MoLC. On the other hand, the MSE of the roughness estimates produced by FAMoLC was lower than MoLC for the scenarios with the highest values of $|\alpha|$. The MSE of the estimates obtained by MoLC remained stable, regardless of the increase of the sample size.

Table 2 presents the average estimation time in seconds for all experiments with the whole scenarios. It shows that FAMoLC performed remarkably faster than MoLC for parameter estimation using synthetic data.

Table 2. The average computation time for parameter estimation using the whole scenarios.

\begin{tabular}{cc}
\hline FMoLC & MLC \\
\hline 6.05 & 94.34 \\
\hline
\end{tabular}

Despite the sensitivity of FAMoLC to the sample size, both estimation methods achieved similar MSE values. Concerning the computational time, FAMoLC decreased markedly in the tests with synthetic SAR data. Here, the experiments confirmed that the fast approach provides a reliable estimation of the roughness and scale parameters of the $G_{I}^{0}$ distribution, and that the fast MoLC can be used in statistical SAR image processing.

\subsection{Experiments with real SAR images and applications}

To evaluate the computational time of FAMoLC and MoLC in real SAR image processing, we designed an experiment where

Figure 3. Mean-Square Error of the scale parameter $(\gamma)$ estimates. The solid line refers to FMoLC and the dot line to

MoLC.

the roughness and scale parameters of the $G_{I}^{0}$ distribution were estimated by using these estimation methods. Inspired by (Mejail et al., 2003), we estimated the parameters for each image pixel by applying a sliding window of $5 \times 5$ pixels and using both estimation methods on real SAR images. Fig. 4 presents multitemporal intensity SAR images acquired by ALOS/PALSAR (L-band, 1 look and HH channel) over the Campos Basin on the Southern Brazilian coast, via two consecutive acquisitions.

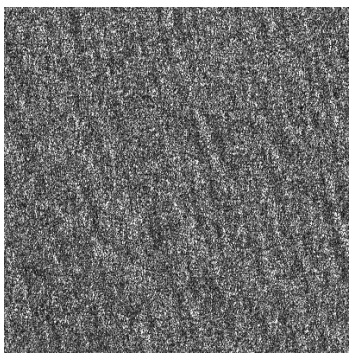

(a)

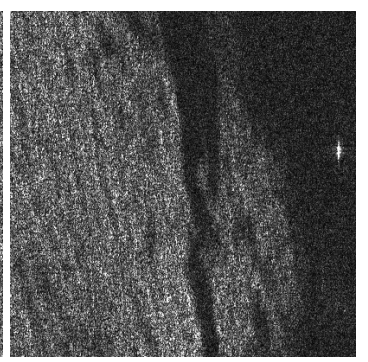

(b)
Figure 4. Multitemporal intensity SAR images acquired by ALOS/PALSAR over the Campos Basin in the Brazilian coast with $\mathrm{HH}$ polarization, single look, size $400 \times 400$. (a) The first acquisition in 05/22/2010 and (b) the second in 08/22/2010.

Table 3 displays the overall computational time to estimate the roughness and scale parameters for each pixel of a real SAR image of size 400x400, as displayed in Fig.4, using both estimation methods. Our approach performed significantly faster than MoLC and hence it is capable of providing reliable inputs (e.g. estimated parameters) for SAR image segmentation, classification and change detection algorithms. 
Table 3. Computational time for parameter estimation for both methods, in seconds, applied to the real images in Fig. 4

\begin{tabular}{cc}
\hline FAMoLC & MoLC \\
\hline 3.76 & 1723.50 \\
\hline
\end{tabular}

We also performed an experiment to measure SAR image contrast and evaluate the performance of both MoLC and FAMLoC in terms of the similarity of the estimates. This experiment was inspired by (Frery et al., 2010) and (Nascimento et al., 2010), in which stochastic distances are applied to SAR data that follows the $G^{0}$ distribution model. In the proposed analysis, a grid of resolution $40 \times 40$ pixels is used, totaling 100 samples $\left(B_{i}\right)$ per image (from top-left $B_{1}$ to right-down $B_{100}$ ), as illustrated in Fig.5. The contrast matrices $\left(C_{m}\right)$ are obtained by computing the Arithmetic-Geometric distance $\left(S_{A G}\right)$, as follows:

$$
C_{m}(i, j)=S_{A G}\left(\left\langle\hat{\alpha}_{i}, \hat{\gamma}_{i}\right\rangle ;\left\langle\hat{\alpha}_{j}, \hat{\gamma}_{j}\right\rangle\right)
$$

with $i, j=1 \ldots 100$.

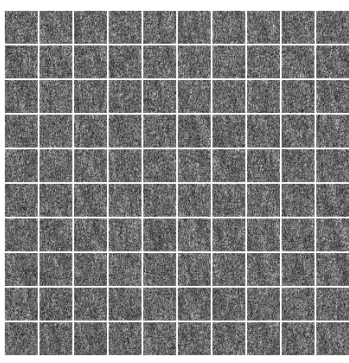

(a)

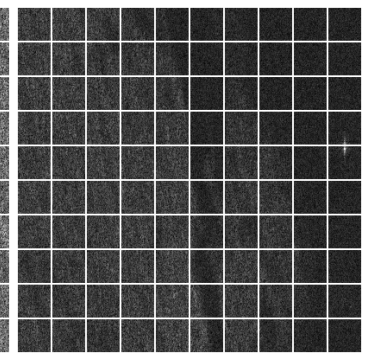

(b)
Figure 5. Grid used to compute the contrast matrices of the SAR images in Fig.4(a) and Fig.4(b).

Regardless of the estimation method for $\alpha$ and $\gamma$, the contrast matrices present similar results. Temporal variations in the scenes are identified by the distinct magnitudes presented in Table 4 .

Table 4. Maximal values of the contrast matrices.

\begin{tabular}{ccc}
\hline & Fig. 5(a) & Fig. 5(b) \\
\hline MoLC & $9.858 \mathrm{e}-01$ & $6.627 \mathrm{e}+01$ \\
\hline FAMoLC & $8.262 \mathrm{e}+01$ & $3.175 \mathrm{e}+03$ \\
\hline
\end{tabular}

\section{CONCLUSIONS}

This paper introduces a fast log-cumulant method that delivers accurate estimates for the roughness and scale parameters of the $G_{I}^{0}$ distribution. Our tests with synthetic SAR data showed that FMoLC performed faster than MoLC, because it did not involve iterative procedures. To test the feasibility of our method in environmental remote sensing applications, we carried out experiments on real multitemporal SAR images of the Campos Basin in Brazil to detect changes on the ocean. The encouraging results from the fast MoLC confirmed that it is computationally suitable to support SAR image processing of large areas and environmental surveillance tasks related to oil spill disasters, for example.

\section{ACKNOWLEDGEMENTS}

This study was supported by CAPES (Finance Code 001), and CNPq (306600/2016-1, 305336/2017-7 and 313887/2018-7).

\section{REFERENCES}

Arfken, G. B., Weber, H. J., 2005. Mathematical Methods for Physicists. Dover Publications.

Bujor, F., Trouvé, E., Valet, L., Nicolas, J.-M., Rudan, J.-P., 2004. Application of Log-Cumulants to the Detection of Spatiotemporal Discontinuities in Multitemporal SAR Images. IEEE Trans. Geosci. Remote Sens., 42(10), 2073-2084.

Frery, A. C., Müller, H.-J., Freitas, C. C., Sant'Anna, S. J. S., 1997. A Model for Extremely Heterogeneous Clutter. IEEE Trans. Geosci. Remote Sens., 35(3), 648-659.

Frery, A. C., Nascimento, A. D. C., Cintra, R. J., 2010. Contrast in speckled imagery with stochastic distances. Proceedings of 2010 IEEE 17th International Conference on Image Processing, IEEE Signal Processing Society, Hong Kong, 69-72.

Gambini, J., Cassetti, J., Frery, A. C., 2015. Parameter Estimation in SAR Imagery using Stochastic Distance an Asymmetric Kernels. IEEE Journal of Selected topics in Applied Earth Observation and Remote Sens., 8(1), 365-375.

Krylov, V., Moser, G., Serpico, S. B., Zerubia, J., 2013. On the Method of Logarithmic Cumulants for Parametric Probability Density Function Estimation. IEEE Trans. on Image Processing, 22(10), 3791-3806.

Lee, J.-S., Pottier, E., 2009. Polarimetric Radar Imaging: from Basics to Applications. CRC Press.

López-Martínez, C., Fàbreagas, X., 2003. Polarimetric SAR Speckle Noise Model. IEEE Trans. Geosci. Remote Sens., 41(10), 2073-2084.

Mejail, M. E., Jacobo-Berlles, J. C., Frery, A. C., 2003. Classification of SAR Images using a General and Tractable Multiplicative Model. International Journal of Remote Sensing, 24(18), 3565-3582.

Nascimento, A. D. C., Cintra, R. J., Frery, A. C., 2010. Hypothesis Testing in Speckle Data With Stochastic Distances. IEEE Trans. on Geosci. and Remote Sens., 48(1), 373-385.

Nicolas, J.-M., 2002. Introduction aux Statistique de Deuxième Espèce: Application des Logs-moments et des Logs-cumulants à l'Analyse des Lois d'Images Radar. Traitement du Signal, 19(3), 139-167.

Rodrigues, F. A. A., Rocha Neto, J. F. S., Marques, R. C. P., Medeiros, F. N., Nobre, J. S., 2016. SAR Image Segmentation Using the Roughness Information. IEEE Geosci. Remote Sens. Lett., 13(2), 146-160.

Singh, J., Datcu, M., 2013. SAR Image Categorization With Log Cumulants of the Fractional Fourier Transform Coefficients. IEEE Trans. Geosci. Remote Sens., 51(12), 5273-5282. 